\title{
L'interleukine 13 : une nouvelle pièce dans le puzzle immunitaire
}

Le réseau des cytokines au sein du système immunitaire est un puzzle dont le nombre de pièces ne cesse d'augmenter, le rendant de plus en plus complexe. Cependant, la découverte d'une pièce manquante peut aussi ouvrir des perspectives nouvelles, permettant de compléter un morceau du puzzle inachevé auparavant. Comment savoir s'il manque encore des pièces et comment les identifier ? Classiquement, la découverte de nouvelles cytokines repose sur l'utilisation d'une activité biologique qui sert de crible, soit pour la purification de la protéine, soit pour le clonage de l'ADNc correspondant, lié à l'expression de la protéine. Mais la redondance des cytokines complique la découverte de nouvelles cytokines de cette façon : une cytokine peut en cacher une autre ! De plus, la nature "pléiotrope " des activités des cytokines fait que les activités principales d'une cytokine sont souvent éloignées de celles utilisées pour sa découverte.

Nous avons donc adopté une stratégie différente, basée, non sur une activité biologique, mais sur des propriétés communes à nombre de cytokines : leur nature inductible et le fait qu'elles soient sécrétées. Dans un premier temps, nous avons organisé et criblé une banque d'ADNc pour rechercher des transcrits des ARNm dont l'expression augmente dans les lymphocytes $\mathrm{T}$ traités par l'anticorps anti-CD28, en costimulation avec un deuxième signal, tel un ester de phorbol ou l'anti-CD2. Cette stimulation, qui mime l'interaction de l'antigène de surface CD28
B7/BB-l des lymphocytes B ou monocytes activés, provoque la prolifération des lymphocytes $\mathrm{T}$ et la production d'un grand nombre de lymphokines [1].

Un des nouveaux ARNm identifié de cette façon, c'est-à-dire dont l'expression est stimulée par l'anticorps anti-(D28, code pour une protéine dont la séquence amino-terminale est composée de façon prédominante d'acides aminés hydrophobes. Cette séquence amino-terminale ressemble donc à un peptide signal, séquence associée à la plupart des protéines sécrétées et clivée lors de leur sécrétion. La protéine codée par cet ARNm remplit nos deux critères pour une cytokine : c'est une protéine inductible et potentiellement sécrétée. Le deuxième critère a été vérifié expérimentalement par l'introduction de l'ADNc, inclus dans un vecteur d'expression, dans les cellules (OOS*. Après marquage des protéines à l'aide de la méthionine ${ }^{35} \mathrm{~S}$, nous avons pu mettre en évidence une protéine sécrétée, de poids moléculaire apparent $9 \mathrm{kDa}$ d'après la migration électrophorétique en gel de polyacrylamide en présence de SDS [2]. Les analyses de masse et de séquence amino-terminale ont révélé que cette protéine comprend, en fait, 112 acides aminés (masse moléculaire 12,5 kDa).

La séquence de cette protéine nous a apporté des renseignements précieux sur sa nature :

(1) La protéine présente une analogie, bien que faible, avec l'IL4 [2], en particulier dans les régions d'hélice- $\alpha$, importantes pour l'activité de l'IL4 $[3,4]$.
(2) Elle présente une forte analogie avec une protéine codée par un ADNc de souris (P600) également isolée par criblage différentiel d'une banque d'ADNc par l'équipe de G Zurawski à DNAX (Palo Alto, CA, USA) $[5,6]$. Bien que le rôle de cette protéine fût à l'époque inconnu, on savait qu'elle était exprimée chez la souris préférentiellement par les lvmphocytes $\mathrm{CD} 4^{+}$de type Th2** [7]. P600 n'est exprimée chez la souris, ni par les lymphocytes Thl**, ni par les lymphocytes T8 cytotoxiques $[7,8]$.

(3) Une autre équipe californienne, celle de Genelabs (Redwood City), a recherché les gènes adjacents à celui de l'IL4 sur un YAC (chromosome artificiel de levure) constitué d'un morceau du chromosome 5 humain contenant les gènes codant pour IL4 et IL5. Par amplification PCR des ADNc s'hybridant avec ce YAC, elle a identifié un gène situé juste en amont du gène codant pour IL4 [9]. Ce gène s'est avéré être celui de l'II.13 [2].

Bien que ne connaissant pas l'activité de notre cytokine, nous savions donc qu'il s'agissait d'une lymphokine de type Th2, faisant partie de ce que W. Paul appelle la "famille

* Cellules de rein de singe exprimant l'antigene $T$ de SV 40, qui permellent l'expression transitoire à un niveau élerie de gènes introduits par transfection.

* Th2 : lymphocytes $T$ auxiliaires ou helper du type 2 qui expriment les II 4,5 et 10 el internennent plus spécialement dans l'immunite humorale. Thl: lymphocyles $T$ auxiliaires ou helper du type 1 qui expriment I'Il.2 et l\% $\% \mathrm{Ng}$ et interviennent plus spécialement dans l'immunité cellulaire. 
II.4" de lymphokines, située sur le bras long du chromosome 5 humain [10]. Comment l'appeler : Nous l'avons baptisée NC.30 (pour nouvelle cytokine 3()). L'équipe de I)NAX avait nommé l'ADNc de souris $\mathrm{P} 60(0)$ parce que l'enzyme de restriction Pstl produisait un fragment de 60) paires de bases ! Après la présentation de notre cytokine ainsi que de celle de l'équipe de DNAX lors du dernier congrès de Keystone sur les cytokines en février 1993, il a été convenu d'appeler cette protéine interleukine 13. Cette terminologie a été adoptée [11] et semble légitime ; la protéine correspond aux règles définies pour une telle molécule : sécrétée par une cellule du système immunitaire et active sur plusieurs cellules du système immunitaire $(\mathrm{m} / \mathrm{s}$ $n^{\circ}$ 6-7, vol. 9, p. 812). C'est ce deuxième point que nous allons aborder maintenant.

I.e lymphocyte B est une des cibles privilégiées des lymphokines produites par les lymphocytes Th2. L'II.13, en accord avec cette attente, exerce de nombreuses activités sur les lymphocytes B chez l'homme, bien que ce ne soit pas le cas chez la souris [11], soulignant la difficulté d'extrapoler entre espèces nos comnaissances d'une crtokine. L'Ill 3 est un facteur de prolifération des lymphocytes $B$ humains, en costimulation avec les anti-immunoglobulines ou l'anti-C.D40 $[6,12$, 13]. Son effet prolifératif est additif à celui de l'II.2 [13], alors que l'II.4 inhibe l'effet de l'Il.2 [14]. Elle module l'expression de la molécule de surface C.D23, mais sur une population de cellules plus restreinte que celle touchée par l'Il.4 [13]. En prolifération également, l'IL.13 agit préférentiellement sur une sous-population des lymphocytes B ; ceux qui sont positifs pour les IgD de surface, correspondant à des cellules vierges [13]. Bien qu'elle puisse augmenter légèrement la production des IgM et $\operatorname{IgG}[6,12]$, elle ne semble pas être un facteur majeur dans la production globale de ces isotypes d'immunoglobulines, comparée à l'Il.2 et à l'll.10 [13]. En revanche, elle stimule nettement la sỵnthèse des $\operatorname{IgG} 4$ et des IgE, propriété que l'on ne comnaissait que pour l'Il.4 $\left(\mathrm{m} / \mathrm{s} n^{\circ} l\right.$, vol 8, p. 83, [12, 13]).

L.'effet stimulateur de l'IL.13 sur la production d'IgE suggère que cette cytokine, au même titre que l'II.4, pourrait jouer un rôle important dans les phénomènes d'allergie. I.'inactivation du gène codant pour l'Il.4 chez la souris a démontré que l'II.4 est nécessaire pour la synthèse d'IgE dans cette espèce, et également pour la génération d'une réponse immunitaire de type Th2 $[15,16]$. Chez l'homme, en revanche, l'activité stimulatrice de la synthèse des IgE produite par des cellules $T$ issues de patients atopiques n'est que partiellement inhibée par un anticorps anti-Il.4 [17]. Nous proposons que l'activité résiduelle soit le fait de l'IIL 3.

Une deuxième cible de l'Il.13 est le monocyte/macrophage. Elle module la morphologie de ces cellules, leur prolifération, l'expression des antigènes de surface, la production de cytokines et leur activité procoagulante $[2,6,18-22]$. L.es activités de l'II.13 sur les molécules de surface des monocytes domnent lieu à un phénotype alternatif de celui induit par l'interféron $\gamma$ ou l'IL.10 [19, 20, 22, 23] (figure 1). Par exemple, les antigènes d'histocompatibilité de classe Il (et la présentation d'antigène) sont augmentés par l'IFN $\gamma$ et l'Il.l 3 et diminués par l'Il.10) les récepteurs $F c$ (et la cytotoxicité dépendante d'anticorps) sont augmentés par l'IFNy et l'Il.l (), et inhibés par l'IL.13 ; et le récepteur mannose (impliqué dans la phagocytose des micro-organismes mannosylés [24]) est augmenté par l'IL.13 et inhibé par l'IFN $\gamma$. I.'IL.13 partage avec l'Il.4 et l'IL.10 un rôle antiinflammatoire en inhibant la production de cytokines, telles l'Ill, l'II.6, l'II.8 et le TNF $\alpha$, en réponse aux lipopolysaccharides bactériens $[2,19]$. L a fonction anti-inflammatoire de l'Il.13 est renforcée par sa capacité d'augmenter la production de deux inhibiteurs de l'Il.l (le récepteur antagoniste de l'Il.l et le récepteur de type II de l'ILl, soluble) $[19,25,26]$. L a synthèse de
l'Il.6 par d'autres types cellulaires (cellules endothéliales, kératinocytes) est, en revanche, augmentée par l'Il.1 3 et l'II.4 [27, 28]. Les effets de l'Il.l 3 in vivo sur la production locale et globale de l'II.6 restent donc à déterminer.

Qu'en est-il du récepteur de l'Il.13: Comme la grande similitude des activités de l'Il.1 3 et de l'II.4 le laissaient supposer, les deux cytokines agissent par un récepteur partagé sur de multiples types cellulaires ([29, 29 bis] ; C. I abit, communication personnelle). Cependant, la sous-unité du récepteur de l'II.4 caractérisée chez la souris et chez l'homme (II.4R- $\alpha$ ), ne lie pas l'II.13, et n'est pas suffisante pour faire un récepteur de forte affinité [29]. Deux publications récentes montrent que la chaîne $\gamma$ du récepteur II.2 participe également au complexe récepteur II.4, et augmente l'affinité de l'IL4 pour son récepteur $[30,31]$. Il semble nécessaire d'envisager au moins trois sous-unités composant les récepteurs de l'Il.4 et de I'Il.1 3 : Il.4R- $\alpha$ liant spécifiquement l'Il.4, une sous-unité $\gamma$ conférant au récepteur une forte affinité pour l'Il.4 et peut-être pour l'II.l 3, et enfin une sous-unité IL.13R- $\alpha$ liant l'II.13 (et peut-être l'II.4).

Certaines activités de l'II.4 ne sont pas partagées par l'II.l3. En effet, l'Il.4 est un facteur de prolifération pour les lymphocytes $T$ alors que I'II.13 n'a pas cet effet [11, 29]. Par ailleurs, l'Il.l 3 n'inhibe pas directement la production d'interféron $\gamma$ induite par l'II.2 dans les lymphocrtes $\mathrm{T}$ et $\mathrm{NK}$, comme le fait l'II.4 ([2, 32, 33], A Minty, résultats non publiés). Les effets de l'IL.13 sur la production de monokines, tel l'Il.12 et le $\mathrm{TNF} \alpha$, qui sont d'importants cofacteurs avec l'Il.2 pour la production de l'interféron $\gamma$ [34], peuvent être inhibiteurs ou activateurs selon le type d'activation monocytaire ([19], A. Minty, résultats non publiés). On peut donc s’attendre à ce que la réponse immunitaire induite par l'II.l3 diffère de celle produite par l'Il.4, qui provoque une réponse humorale, du type Th2, et inhibe la réponse d'immunité cel- 
lulaire de type Th1 [16, 35]. Cette absence de réponse cellulaire induite par l'IL4 est délétère dans de nombreuses situations pathologiques [35] (voir ci-dessus).

Plusieurs possibilités d'applications cliniques s'ouvrent pour l'IL13. Son activité anti-inflammatoire pourrait s'excercer dans le contexte des maladies inflammatoires. Pour les maladies inflammatoires aiguës, tel le choc septique, l'activité anti-inflammatoire plus forte de l'ILl0 serait peut-être plus avantageuse [36]. C'est dans la résolution de l'inflammation chronique, constatée dans certaines maladies auto-immunes, que l'on recherchera une application pour l'ILl 3. En effet, l'IL13 diminue la sévérité de la maladie dans les modèles d'arthrite rhumatoĩde et d'encéphalomyélite expérimentale chez le rat [36 bis] (C. Fournier, D. Fradelizi, communication personnelle).

En tant qu'immunomodulateur, l'IL13 pourrait avoir une action antitumorale, car l'IL13 exerce ce type d'activité dans deux modèles expérimentaux chez la souris (S. LebelBinay et D. Fradelizi, communication personnelle). Dans le premier, la co-injection aux souris nude de cellules HeLa avec des cellules $\mathrm{CHO}$ exprimant l'ILl3 empêche la formation de tumeur $\left(m / s n^{\circ} 7\right.$, vol. 5, p. 522). Chez ces souris dépourvues de lymphocytes $\mathrm{T}$, on observe donc un rejet tumoral. Les cellules impliquées ne sont pas encore connues. Pour l'IL4, qui présente également un effet antitumoral, un rôle des éosinophiles et/ou des macrophages a été proposé [37, 38]. Dans le second modèle, l'injection de cellules de mastocytome P815 exprimant l'IL13 à des souris immunocompétentes $\mathrm{DBA} / 2$ donne lieu à des tumeurs de plus petite taille que celles constatées avec les cellules P815 parentales. Au bout de 15 jours, ces tumeurs régressent. Les souris ayant reçu une injection de P815-IL13 peuvent recevoir, deux mois plus tard, une nouvelle injection de cellules P815 parentales sans développer de tumeur.

L'IL13 exerce également un effet antiviral. La production du virus VIH-1 dans les cultures de macro-

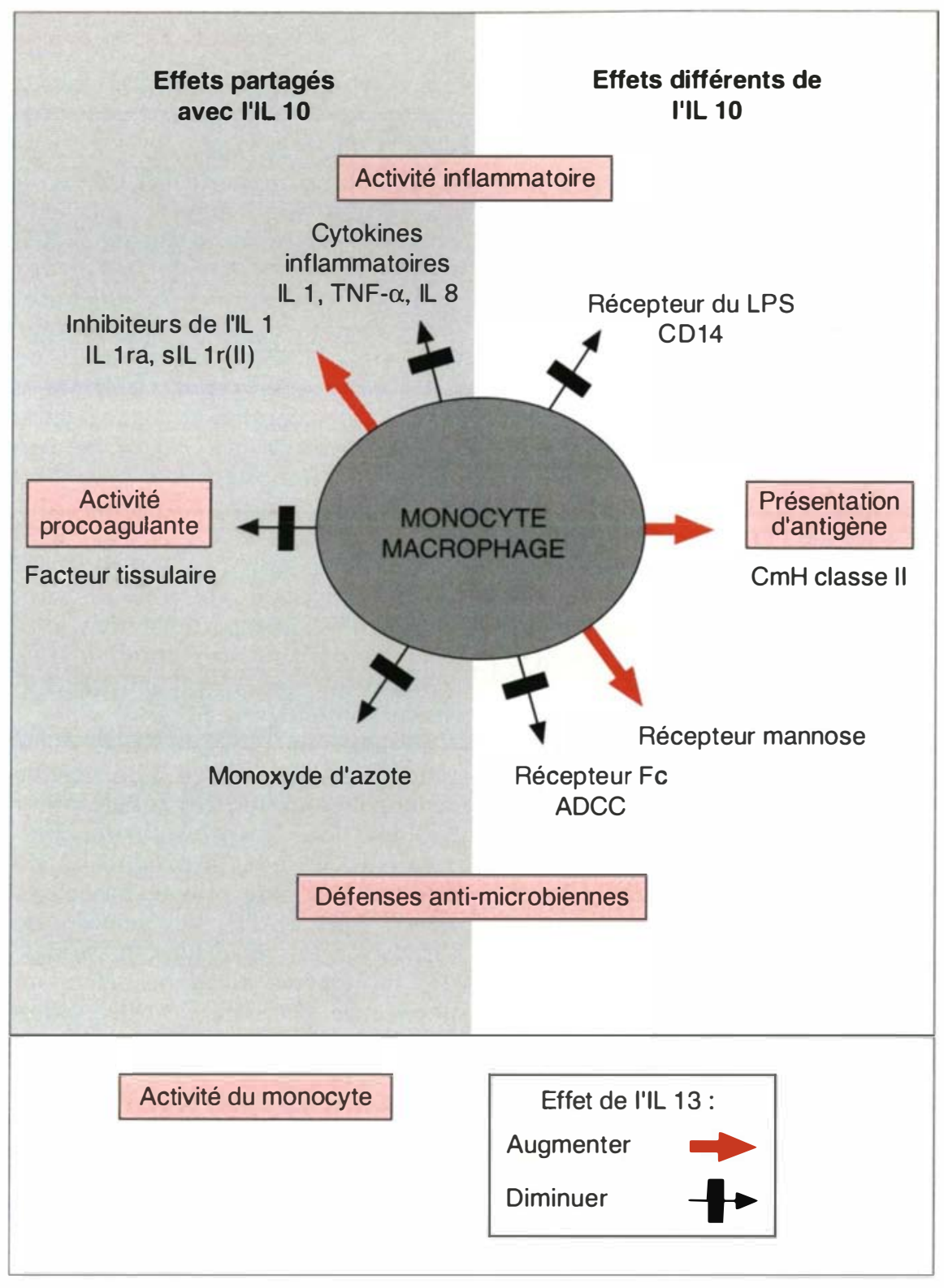

Figure 1. Activités de l'IL13 sur les monocytes et les macrophages. Toutes ces activités sont partagées avec I'IL4 [2, 18-26, 44]. Les activités partagées avec I'IL 10 sont indiquées à gauche sur la figure, alors que celles qui diffèrent sont indiquées à droite [2, 18-23]. Parmi les activités qui diffèrent entre I'IL 13 et I'IL 10 : I'expression du CD14 et du récepteur mannose n'est pas affectée par I'IL 10, alors qu'elle est, respectivement, inhibée et augmentée par I'IL13; I'expression des récepteurs Fc est stimulée par I'IL10 et inhibée par I'IL13, et celle des antigènes d'histocompatibilité de classe II est inhibée par I'IL10 et augmentée par I'IL13. L'effet de I'IL13 sur la production de l'oxyde nitrique peut varier selon le type de macrophage étudié [20, 22]. $A D C C=$ cytotoxicité dépendante d'anticorps. 
phages infectées in vitro est inhibée par l'IL13, alors que l'IL13 est sans effet sur la production du virus par les lymphocytes $T$ [39]. Le macrophage semblant être un réservoir important du virus VIH-1 [40], cet effet virostatique pourrait aider à réduire la charge virale chez les malades atteints du SIDA. Il est à noter que l'IL4, qui peut également inhiber la production du VIH-1 par les macrophages, augmenterait la production de virus par les lymphocytes $T$ ([41], L. Montaner et S. Gordon, communication personnelle). De plus, l'effet de l'IL4 sur le système immunitaire, favorisant une réponse humorale de type Th2 aux dépens d'une réponse Thl d'immunité cellulaire, pourrait, d'après certains auteurs [42], rendre cette cytokine inappropriée pour le traitement du SIDA.

D'autres actions de l'IL13 sur les monocytes la rendent intéressante dans le contexte du SIDA. Son inhibition de la production de l'IL6 et du TNF $\alpha$ devrait avoir pour effet de maintenir la latence du virus dans les macrophages déjà infectés, puisque ces deux cytokines stimulent la production du virus [43]. De plus, l'IL13 augmente la concentration de récepteur mannose sur les macrophages [22]. Ce récepteur a été impliqué dans la phagocytose des micro-organismes mannosylés tels Pneumocystis carinii [24]. L'IL13 pourrait donc diminuer le taux important de mortalité dû à ce type d'agent infectieux chez les malades atteints du SIDA.

En conclusion, la découverte de l'IL13 semble importante sur deux plans. Sur le plan fondamental, elle renforce nos connaissances des mécanismes immunitaires impliqués dans les réactions inflammatoires, humorales et allergiques. Sur le plan clinique, son profil anti-inflammatoire, antitumoral, et antiviral ressemble à celui de l'IL4 [44], mais avec un spectre d'activités plus limité qui pourrait favoriser l'utilisation de l'IL13 Pour l'instant, nous sommes encore loin, sans doute, d'avoir compris toute la signification de cette nouvelle pièce du puzzle immunitaire

\section{REFERENCES}

1. Thompson CB, Lindsten T, Ledbetter JA, Kunkel SL, Young HA, Emerson SG, Leiden JM, June CH. CD28 activation pathway regulates the production of multiple T-cell-derived lymphokines/cytokines. Proc Natl Acad Sci USA $1989 ; 86: 1333-7$.

2. Minty A, Chalon P, Derocq JM, Dumont X, Guillemot JC, Kaghad M, Labit C, Leplatois P, Liauzun P, Miloux B, Minty C, Casellas P, Loison G, Lupker J, Shire D, Ferrara P, Caput D. Interleukin-13 is a new human lymphokine regulating inflammatory and immune responses. Nature $1993 ; 362: 248-50$.

3. Moss BW, Leder P. A receptor binding domain of mouse Interleukin-4 defined by a solid-phase binding assay and in vitro mutagenesis. J Biol Chem 1992 ; 267 : $11957-$ 63

4. Kruse N, Shen BJ, Arnold S, Tony HP, Müller T, Sebald W. Two distinct functional sites of human interleukin 4 are identified by variants impaired in either receptor binding or receptor activation. EMBO J $1993 ; 12: 5121-9$.

5. Brown KD, Zurawski SM, Mosmann TR Zurawski G. A family of small inducible proteins secreted by leukocytes are members of a new superfamily that includes leukocyte and fibroblast-derived inflammatory agents, growth factors, and indicators of various activation processes. $J$ Immunol $1989 ; 142: 679-87$.

6. McKenzie AN, Culpepper JA, de Waal Malefyt RB, Punnonen J, Aversa G, Sato A, Dang W, Cocks BG, Menon S, et al. Interleukin-13, a T-cell-derived cytokine that regulates human monocyte and B-cell function. Proc Natl Acad Sci USA 1993 ; 90 : 3735-9.

7. Cherwinski HM, Schumacher JH, Brown KD, Mosmann TR. Two types of mouse helper T cell clone III. Further differences in lymphokine synthesis between Th1 and Th2 clones revealed by RNA hybridization, functionally monospecific bioassays, and monoclonal antibodies. J Exp Med 1987 ; 166 : 122944 .

8. Fong TA, Mosmann TR. Alloreactive murine $\mathrm{CD} 8^{+} \mathrm{T}$ cell clones secrete the Th1 pattern of cytokines. J Immunol $1990 ; 144$ : 1744-52.

9. Morgan JG, Dolganov GM, Robbins SE Hinton LM, Lovett $M$. The selective isolation of novel cDNAs encoded by the regions surrounding the human interleukin -4 and -5 genes. Nucleic Acids Res $1992 ; 20: 5173-9$.

10. Boulay JM, Paul WE. The interleukin-4 related lymphokines and their binding to hematopoietin receptors. J Biol Chem 1992 . $267: 20525-8$.

11. Zurawski G, de Vries JE. Interleukin-13, an interleukin-4 like cytokine that acts on monocytes and $B$ cells but not on $T$ cells. Immunol Today 1994 ; 15 : 19-26.

12. Punnonen J, Aversa G, Cocks BG McKenzie AN, Menon S, Zurawski G, de
Waal Malefyt R, de Vries JE. Interleukin-13 induces interleukin-4-independent IgG4 and IgE synthesis and CD23 expression by human B cells. Proc Natl Acad Sa USA 1993; $90: 3730-4$

13. Defrance T, Carayon P, Billian G, Guillemot JC, Minty A, Caput D, Ferrara P. Interleukin-13 is a B cell stimulating factor. JExp Med 1994 ; 179 : 135-43.

14. Defrance T, Vanbervliet B, Aubry JP, Banchereau J. Interleukin-4 inhibits the proliferation but not the differentiation of activated human B cells in response to interleukin-2. J Exp Med 1988 ; 168 : 132137.

15. Kuhn R, Rajewsky K, Muller W. Generation and analysis of interleukin-4 deficient mice. Science $1991 ; 254$ : 707-10.

16. Kopf M, Le Gros G, Bachmann M, Lamers MC, Bluethmann H, Kohler G. Disruption of the murine IL-4 gene blocks Th-2 cytokine responses. Nature $1993 ; 362$ : 245-8.

17. Zhang X, Polla B, Hauser C, Zubler RH. $T$ cells from atopic individuals produce IgEinducing activity incompletely blocked by anti-interleukin-4 antibody. Eur I Immunol $1992 ; 22$ : 829-33.

18. Herbert JM, Savi P, Laplace MC, Lale A, Dol F, Dumas A, Labit C, Minty A. IL-4 and IL-13 exhibit comparable abilities to reduce pyrogen-induced expression of procoagulant activity in endothelial cells and monocytes. FEBS Lett 1993 ; 328 : 26870 .

19. de Waal Malefyt R, Figdor CG, Huijbens R, Mohan-Peterson S, Bennett B, Culpepper J, Dang W, Zurawski G, de Vries JE. Effects of IL13 on phenotype, cytokine production, and cytotoxic function of human monocytes. $J$ Immunol $1993 ; 151: 6370-81$.

20. Doherty TM, Kastelein R, Menon S, Andrade S, Coffman RL. Modulation of murine macrophage function by IL-13. I Immunol 1993 ; 151 : 7151-60.

21. Minty A, Chalon P, Guillemot JC, Kaghad M, Liauzun P, Magazin M, Miloux B, Minty C, Ramond P, Vita N, Lupker J, Shire D, Ferrara P, Caput D. Molecular cloning of the MCP-3 chemokine gene and regulation of its expression. Eur Cytokine Netw 1993 ; 4 : 99-110.

22. Doyle A, Herbein G, Montaner LJ Minty AJ, Caput D, Ferrara P, Gordon S. Interleukin-13 alters the activation state of murine macrophages in vitro : comparison with interleukin- 4 and interferon- $\gamma$. Eur I Immunol $1994 ; 24$ : 1441-5.

23. Te Velde $A$, de Waal Malefijt $R$, Huijbens R, de Vries J, Figdor C. IL-10 stimulates monocyte $F c \gamma R$ surface expression and cytotoxic activity. J Immunol $1992 ; 149: 4048-52$.

24. Ezekovitz RAB, Williams D, Koziel $\mathrm{H}$ Armstrong M, Warner A, Richards FF, Rose R. Pneumocystis carinii uptake by alveolar macrophages is mediated via the mannose receptor. Nature $1991 ; 351: 155$. 


\section{REFERENCES}

25. Colotta F, Re F, Muzio M, Bertini R, Polentarutti N, Minty A, Caput D, Ferrara P, Mantovani A. Interleukin-13 induces expression and release of interleukin-1 decoy receptor in human polymorphonuclear cells. J Biol Chem 1994; 269 : 12403-6.

26. Muzio M, Re F, Sironi M, Polentarutti N, Minty A, Caput D, Ferrara P, Mantovani A, Colotta F. Interleukin-13 induces the production of interleukin-l receptor antagonist (IL-1 $\mathrm{r} \alpha$ ) and the expression of the mRNA for the intracellular (keratinocvie) form of IL-1 $\mathrm{r} \alpha$ in human mvelomonocrtic cells. Blood $1994 ; 83: 1738$ 43.

27. Derocg JM, Segui M, Poinot-Chazel C, Minty A, Caput D, Ferrara P, Casellas $P$. Interleukin-13 stimulates interleukin-6 production by human keratinocytes. Similarity with interleukin-4. FEBS Lett 1994 ; $343: 32-6$

28. Sironi M, Sciacca FL, Matteucci C, Conni M, Vecchi A, Bernasconi S, Minty A, Caput D, Ferrara P, Colotta F, Mantovani A. Regulation of endothelial and mesothelial cell function by interleukin-13 : selective induction of vascular cell adhesion molecule-1 and amplification of interleukin6 production. Blood 1994; (sous presse).

29. Zurawski SM, Vega F, Huyghe B, Zurawski G. Receptors for interleukin-13 and interleukin-4 are complex and share a novel component that functions in signal transduction. $E M B O J 1993 ; 12: 2663-70$.

29 bis. Vita N, Lefort S, Laurent P, Caput D, Ferrara $P$. The interleukin-13 receptor: characterisation and comparison with the interleukin-4 receptor on several cell types. J Biol Chem 1994 (sous presse).

30. Kondo M, Takeshita T, Ishii N, Nakamura M, Watanabe S, Arai K, Sugamura K. Sharing of the interleukin-2 (IL-2) receptor $\gamma$ chain between receptors for IL-2 and IL-4. Science $1993 ; 262$ : $1874-$ 83.

31. Russell SM, Keegan AD, Harada N, Nakamura Y', Noguchi M, Leland P, Friedman MC, Mivajima A, Puri RK, Paul $\mathrm{W}^{\mathrm{E}}$, Leonard WJ. Interleukin-2 receptor $\gamma$ chain a functional component of the interleukin-4 receptor. Science $1993 ; 262$ : 1880-3.

32. Tanaka T, Hu-LI J, Seder RA, Fazekas de St. Groth B, Paul WE. Interleukin-4 suppresses interleukin-2 and interferon $\gamma$ production by naive $T$ cells stimulated by accessory cell-dependent receptor engagement. Proc Nall Acad Sci USA 1993 ; $90: 5914-8$

33. Erard F, Wild M-T, Garcia-Sanz JA, Le Gros G. Switch of CD8 T cells to noncytolytic CD8 CD4 cells that make Th2 cytokines and help B cells. Science 1993 ; $260: 1802-5$.

34. D'Andrea A, Aste-Amezaga M, Valiente NM Ma X, Kubin M, Trinchieri G. Interleukin-10 inhibits human lymphocyte interferon $\gamma$ production by suppressing natural killer cell stimulatory factor/1L-12 svnthesis in accessory cells. J Exp Med $1993 ; 178$ : 1041-8.
35. Leal LMCC, Moss DW', Kuhn R, Muller W Liew FY. Interleukin-4 transgenic mice of resistant background are susceptible to Leishmania major infection. Eur Immunol $1993 ; 23: 566-9$

36. Goldman M, Velu T. L'interleukine-10, une nouvelle cytokine immunosuppressive et anti-inflammatoire. médecine/sciences $1993 ; 9: 453-5$.

36 bis. Cash E, Minty A, Ferrara P, Caput D, Fradelizi D, Rott O. Macrophage inactivating interleukin-13 suppresses experimental autoimmune encephalomyelitis in rats. J Immunol 1994 (sous presse).

37. Tepper RI, Coffman RI, Leder P. An eosinophil-dependent mechanism for the antitumor effect of interleukin-4. Srience $1992 ; 257: 548-51$.

38. Golumbek PT, Lazenby AU, Levitsky HI Jaffee LM, Karasusama H, Baker M, Pardoll DM. Treatunent of established renal cancer by tumor cells engineered to secrete interleukin4. Science $1991 ; 254$ : 7136

39. Montaner LJ, Doyle AG, Collin M, Herbein G, Illei $P$, James $W^{\prime}$, Minty $A$ Caput D, Ferrara P, Gordon S. Interleukin-13 inhibits human immunodeficiency virus type 1 production in primary blood-derived human macrophages in vitro. J Exp Med $1993 ; 178: 743-7$.

40. Weiss RA. How does HIV cause AIDS ? Science $1993 ; 260$ : $1273-9$

41. Schuitemaker H, Koostra MHG, Koppelman SM, Bruisten HG, Huisman M Tersmette M, Miedma F. Proliferation dependent HIV-1 infection of monocytes occurs during differentiation into macrophages. J Clin Invest 1992;89: 1154 60.

42. Cohen J. T cell shift : ker to AIDS therapy? Srience $1993 ; 262: 175-6$.

43. Fauci AS. Multifactorial nature of human immunodeficiency virus disease implications for therapv. Science 1993 ; 262 $1011-8$

44. Banchereau J. Interleukine-4. médecine/ sciences $1990 ; 6: 946-53$.

\section{Adrian Minty}

Sanofi Recherche, Labège Innopole, voie $n^{\circ}$ 1, BP137, 31328 Labège Cedex, France.

\section{TIRÉS A PART}

\section{A. Mints:}

